\title{
Using personal digital assistants (PDAs) for the collection of safety belt use data in the field
}

\author{
JONATHON M. VIVODA and DAVID W. EBY \\ University of Michigan Transportation Research Institute, Ann Arbor, Michigan
}

\begin{abstract}
From 1975 through 2003, because of the use of safety belts, an estimated 180,000 deaths from traffic crashes have been prevented. In order to assess the gains that have been made in safety belt use across the U.S., in individual states, and in communities, belt use surveys are conducted at regular intervals to determine use rates. The most valid method for surveying safety belt use is through direct observation. Direct observation surveys are conducted along roadways by trained researchers looking into passing vehicles and recording safety belt use. This method of data collection has been effective in the past through the use of paper-and-pencil data recording, yet it could be improved through the use of electronic and communication technology. Reported here is a study designed to compare electronic data collection, using personal digital assistants (PDAs), with collection using the traditional paperand-pencil method during the annual statewide survey of safety belt use in Michigan that we have been conducting since 1984. The goals of the study were to develop a PDA database program for data entry in the field, to directly compare the PDA data collection process with the paper-and-pencil method on both accuracy and speed, and to assess mechanical and environmental factors, such as battery life, screen visibility, and reaction to adverse weather, that may act as limitations to the PDA method, in comparison with the paper-and-pencil method. In a direct comparison of methods, two observers collected data at the same roadway intersections, one using paper and a pencil and one using a PDA equipped with our custom software. The study showed that the PDA method was as fast and as accurate as the paper-and-pencil method. There were no adverse effects on the PDA caused by environmental conditions. The PDA was superior to the paper-and-pencil method in rainy weather and for data collector supervision. In addition, the use of the PDA obviated the need for entry of paper-recorded data into an electronic format. We conclude that the use of PDAs for safety belt field data collection is superior to the paper-and-pencil method. A Web address is given where the custom PDA safety belt data collection software can be obtained free of charge.
\end{abstract}

The rate of safety belt use in the United States is currently about $79 \%$. This is the highest use rate ever observed for the nation as a whole (Glassbrenner, 2003). From 1975 through 2003, it is estimated that the use of safety belts has prevented nearly 180,000 deaths (Glassbrenner, 2003; National Highway Traffic Safety Administration [NHTSA], 2002). In order to assess the gains that have been made in safety belt use across the U.S. and in individual states, belt use surveys are conducted at regular intervals to determine use rates. These surveys allow traffic safety professionals, legislators, and the public to understand changes and trends in overall safety belt use. These surveys can also determine safety belt use rates by various groups within the driving population. For example, nearly every safety belt survey conducted in the U.S. has shown that male motorists buckle up less often than females and pickup truck occupants use safety belts less often than do motorists in other vehicle types (see, e.g., Bondy \& Glassbrenner, 2001; Eby, Molnar, \& Olk, 2000; Eby, Vivoda, \& Fordyce, 2002). As belt use across

Correspondence concerning this article should be addressed to J. M. Vivoda, UMTRI-SBA, 2901 Baxter Rd., Ann Arbor, MI 48109-2150 (e-mail: jviv@umich.edu). the U.S. continues to rise, it becomes even more important to measure safety belt use by the various demographic and vehicle characteristics. Understanding which groups use safety belts less often allows traffic safety experts to plan safety belt promotion campaigns designed to reach these low belt use groups. Increasing belt use among these groups is of paramount importance for increasing overall safety belt use across the U.S.

There are only three methods for assessing safety belt use rates: analysis of crash records that indicate belt use; self-reported methods, such as a telephone interview; and direct observation surveys in which belt use is recorded along roadways. Each of these methods has both advantages and disadvantages. Analysis of crash records is quite inexpensive and allows researchers to link belt use and injury severity (S. Kim \& K. Kim, 2003; Yau, 2004). Unfortunately, belt use in these records is often self-reported by the driver to a police officer at a crash scene. Since lack of belt use is illegal in all but one state, drivers are reluctant to admit such a violation when asked, which leads to crash records that are biased toward higher belt use (K. Kim, 1999). Even when use is honestly reported, those who use belts may be less likely to be crash involved, again leading to a bias in use rates calculated from crash records. Selfreported methods, such as telephone surveys, are easily 
administered and allow for detailed information gathering on safety belt use and lack of use. For example, it is possible to ask phone participants about their income, education, and the reasons why they fail to wear a safety belt. However, self-reported safety belt use has been shown to be inaccurate, with respondents tending to overreport their safety belt use (see, e.g., Streff \& Wagenaar, 1989).

The most accurate method for measuring safety belt use is the direct observation survey. In fact, this is the only survey method that NHTSA will accept when a state reports statewide belt use in order to qualify for federal traffic safety programs (NHTSA, 1998). Direct observation surveys are conducted along roadways by trained researchers looking into passing vehicles and recording safety belt use. Since this method does not require interaction with the motorists and the motorists are unaware of the researcher's purpose near the roadway, an accurate assessment of safety belt use is possible. The disadvantage of this method is that only observable variables that may be related to belt use, such as occupant sex, age group, seating position, and vehicle type, can be collected at the same time as the belt use data. One cannot assess level of income or education, which may influence the use of belts greatly.

Direct observation surveys have been used in the U.S. to assess safety belt use since the early to mid-1980s, when the first statewide mandatory safety belt laws were implemented. Currently, nearly every state conducts an annual direct observation safety belt survey. NHTSA also conducts a direct observation survey of safety belt use every 2 years, called the National Occupant Protection Use Survey (NOPUS), for which a nationwide sample is used (see, e.g., Glassbrenner, 2003; NHTSA, 2001). Historically, these studies have been conducted using a paperand-pencil or mechanical counter method. As vehicles pass researchers, safety belt use, demographic information, and vehicle type is typically recorded on precoded observation forms, or a traffic counter (clicker) is used to count the numbers of belted and total occupants. This method of data collection has been effective in the past, yet it could be improved through the use of electronic and communication technology. For example, data collected using paperand-pencil methods have to be converted to an electronic format prior to data analysis. This is typically done using computer scanning technology or expensive human data entry. In many cases, the human data entry process can take weeks, and errors can be introduced during the process. In many states and jurisdictions, quick turnaround and short project deadlines have created a need for quick results that cannot wait for the time-consuming data entry process.

To address these limitations, we conducted a study designed to compare electronic data collection using personal digital assistants (PDAs) with the traditional paperand-pencil method during the annual statewide survey of safety belt use in Michigan that we have been conducting since 1984 (Eby et al., 2000; Eby et al., 2002). Using PDAs for data collection eliminates both the time necessary for data entry and the errors that can be introduced during the process. The goals of the study were to develop a PDA database program for data entry in the field, to directly compare the PDA data collection process with the paper-and-pencil method on both accuracy and speed, and to assess mechanical and environmental factors, such as battery life, screen visibility, and reaction to adverse weather, that may act as limitations to the PDA method, in comparison with the paper-and-pencil method.

\section{METHOD}

\section{Hardware}

Before the study began, several different PDA models were researched and considered. In order to maximize the efficiency of safety belt data collection using this technology, it was important that the PDA used for the study should have several key characteristics. To facilitate communication, ease of data entry, and speed of data transmission, Palm Tungsten W PDAs using the Palm operating system platform were chosen. These PDAs have the builtin capabilities of a cellular phone, wireless Internet, a small keyboard, and $16 \mathrm{MB}$ (megabytes) of memory. The Tungsten $\mathrm{W}$ also has a 16-bit, $320 \times 320$ color display that supports over 65,000 colors. In addition, the Tungsten W has an expansion card slot for Secure Digital (SD) cards, allowing for near unlimited data storage. At the time of the study, this was the only Palm-based PDA with all of these capabilities.

\section{Software Development}

The study was designed to directly compare the use of PDAs with that of the paper-and-pencil method of data col-

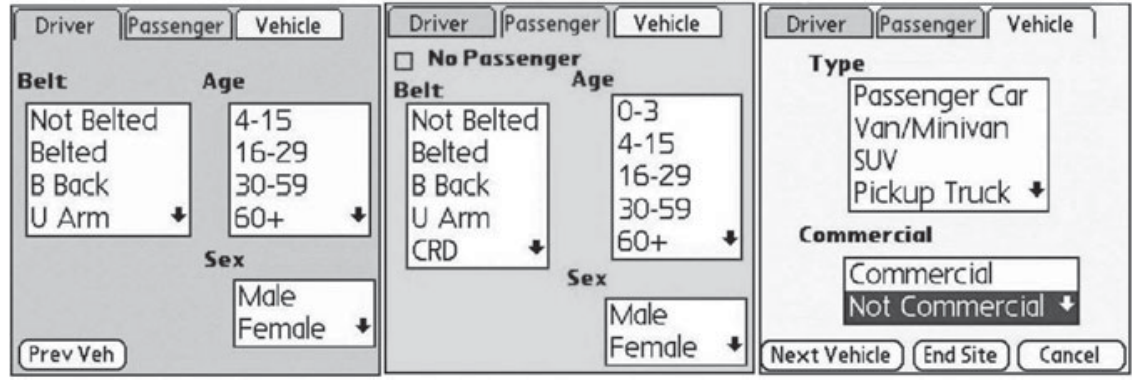

Figure 1. Electronic observation form screens. 


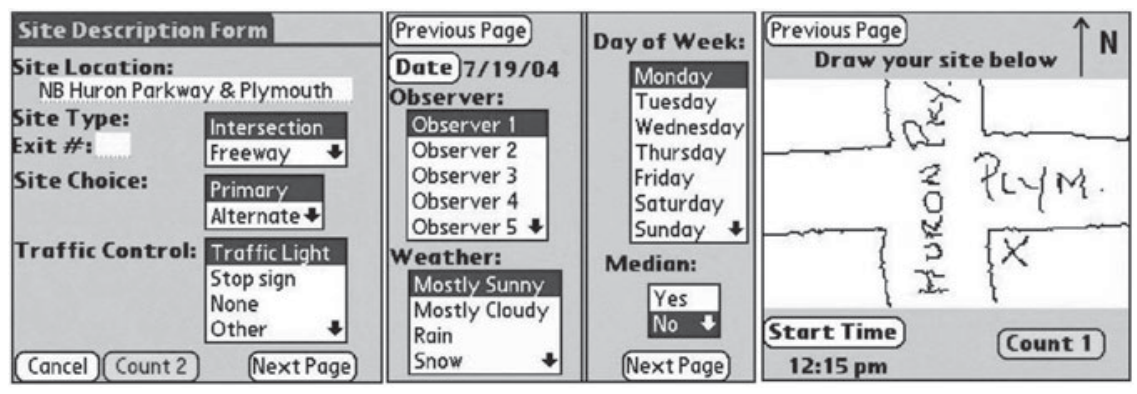

Figure 2. Electronic site description form screens.

lection. Since safety belt data collection is a new use for PDAs, it was necessary to develop custom electronic PDA forms for data entry. After the functionality of several programs had been investigated, HanDBase was chosen as the best database software for this purpose. ${ }^{1}$ Two sets of forms were developed: an observation form, used for collecting observed safety belt use data, motorist demographics, and vehicle information, and a site description form for recording information about the site location, weather conditions, day of week, time of day, traffic control, and so on. Figure 1 shows an example of the observation form data entry screens. As is shown, the observation form was divided into separate color-coded screens containing information about the driver, front-right passenger, and vehicle. Each screen was accessible by tapping with a stylus on the appropriate tab across the top of the screen. Within each screen, each category of data and the choices for each category were displayed. Researchers tapped the screen to select the proper safety belt use and demographic information for the driver and front-right passenger. The selected data then appeared highlighted on the screen. When new data were entered, the first screen that appeared was one for entering driver information, followed by front-right passenger information and, finally, vehicle information. As Figure 1 shows, "Not Commercial" was selected as a default for vehicle type, since this selection applies to most vehicles. If a vehicle was commercial, the researchers could denote this by tapping on it.

Figure 2 shows the electronic site description form. The data entry format for this form was very similar to the observation form. The site location (street names and standing location) was typed in using the PDA's small built-in keyboard, whereas the remaining data were entered following the same procedures as those described for the observation form. Additional site and environmental information, as well as the date, a sketch of the intersection, and the site's start time, was entered on subsequent screens.

The electronic site description form also allowed the researchers to conduct traffic counts without resorting to the use of a mechanical traffic counter. As is shown in Figure 3, a built-in traffic counter was used to count vehicles both before and after data collection. ${ }^{2}$ To use the electronic counter, the researchers tapped on the large "+ " button to increment the count display at the top. The size of this button allowed the researcher to tap the screen while watching the roadway.
If a mistake was made, the researcher could decrease the count by tapping on the small "- " button. The last screen of the site description form (also shown in Figure 3) allowed the researcher to enter the end time of the data collection at the site, the number of minutes of interruption (if any), and any comments regarding the site or traffic flow that may have been important.

\section{Procedure}

Data collection was scheduled to coincide with the statewide safety belt survey that we conduct annually in the fall in Michigan with four field researchers. To assess the accuracy of the data collected using a PDA, in comparison with the paper-and-pencil method, an additional researcher was hired. This researcher (referred to here as the PDA researcher) had a long history of using the paperand-pencil method in our previous safety belt use studies. The data from the PDA researcher, who used the PDA data collection method, are compared with the data collected by another researcher, who used the traditional paper-andpencil method. Both researchers collected data at the same roadway intersections and at the same times. Prior to data collection, all the researchers participated in several days of intensive training, including both classroom review and practice field observations. All the researchers attained an interresearcher reliability level ${ }^{3}$ of at least $85 \%$ on each category of data collected. Throughout the data collection, the project supervisor also performed unannounced site checks, to ensure quality data collection by all of the researchers.

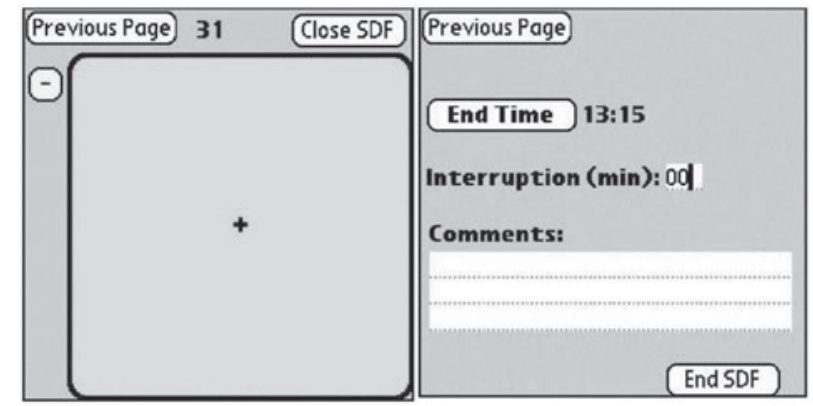

Figure 3. Electronic counter and final site description form screen. 


\section{Practical Testing of the PDA for Use in the Field}

The study also assessed potential mechanical and environmental limitations to the PDA method, in comparison with the paper-and-pencil method. These issues were assessed throughout the survey by the PDA researcher and the field supervisor, during normal everyday use. The mechanical factors investigated were battery life, data storage capacity, data transfer (e-mailing data), contingencies for failure or breakage of PDA in the field, and general ease of use.

To provide a contingency for battery life problems in the field, we provided the PDA researcher with a car charger and a plug-in home charger. To expand the storage capacity of the PDA, the researcher was also provided with a 64-MB removable SD card. After the researcher had transferred data to the project supervisor, the site data were moved from the internal PDA memory to the SD card.

Another important aspect of PDA use that was tested during this study was related to how the data were transferred from the PDA researcher to the project supervisor. To take advantage of the built-in wireless e-mail capabilities of the PDA, an e-mail account was configured for the PDA prior to the beginning of data collection. The PDA researcher was instructed to e-mail data to the project supervisor at breaks throughout the day (usually during a lunch break and, again, at the end of the day). Upon completion of data collection, the researcher opened the e-mail program on the PDA and composed an e-mail to the project supervisor, with the data collection files as an attachment. During the study, the researcher tested the ease of use of the program, as well as issues related to the size of the attachments and cellular roaming.

The effect of various weather conditions on PDA use was also tested during this study. In particular, we were concerned with the possible negative effects of rain and cold weather on the PDA. To eliminate potential problems associated with water damage to the PDA during wet weather, the PDA was placed inside a plastic freezer bag (for food) that could be zipped closed, making it nearly impossible for the PDA to get wet. The PDA researcher also tested the PDA in various lighting conditions in order to assess the visibility of the PDA screen. It was more difficult to see the screen during extremely sunny conditions, but counterintuitively, we found that if the PDA screen was turned toward the sunlight (rather than away from it), it was easier to see. However, the PDA screen was relatively easy to see with all lighting conditions.

To prepare for possible PDA failure or breakage, we purchased a backup PDA for the study as a contingency plan. Since it would not be possible to deliver the backup PDA to the researcher during a distant overnight trip, we also provided paper data forms for the PDA researcher.

\section{RESULTS}

As was described earlier, before making the change from paper-and-pencil data collection to PDA data collection, there were important issues that needed to be addressed and submitted to tests. These issues can be categorized into three main areas: accuracy, speed, and mechanical/environmental issues. The results of scientific tests that were conducted in order to compare the accuracy and speed of the two data collection methods will be discussed here, and the anecdotal results related to mechani$\mathrm{cal} /$ environmental issues, as well as a cost analysis, will be outlined in the Discussion section.

\section{Accuracy}

The most important issue to be addressed was accuracy. If there were to be something inherently different about collecting data with a PDA that affected the accuracy of the collected data, the PDA method would not be viable for the collection of safety belt use field data. To compare the data collected by the two researchers, the relative contribution of each variable to its category was assessed. By examining the data in this way, it was possible to eliminate differences in the data due to differing speeds of data collection between the two researchers. Since the researchers were collecting data at the same roadway intersections, even if one researcher collected more data than did the other, the proportion of belted occupants (and all other variable comparisons) should be about the same for both researchers, if the two methods of data collection do not differ. Table 1 shows a comparison between the two methods according to the proportions of each category of data collected, along with the associated statistics. Included in this table are the comparisons according to proportions of safety belt use, sex, age group, and vehicle type. Within each category, chi-square analyses revealed that the differences in the proportions of these groups were not statistically significant. These results illustrate that the PDA and paper-and-pencil data collection methods are comparable to each other with regard to accuracy; that is, the accuracy of data collection is not affected by the PDA data collection method.

\section{Speed}

Speed (or volume) of data collection using a PDA was also assessed during the study. As was mentioned previously, comparing the amount of data collected by one researcher with the amount collected by another can raise problems, because researchers naturally collect data at different speeds. To take this problem into account, we assigned the PDA researcher to "Researcher Schedule 1". ${ }^{4}$ This particular researcher was hired to be the PDA researcher because he had been assigned to this schedule when the paper-and-pencil method had been used during the previous two fall surveys. Thus, a comparison was possible between the data from the PDA researcher during the present study and his own paper-and-pencil data from the previous 2 years at the same site locations, during the same times of day, days of week, and time of year. There were a few site changes during this 3-year period, but these sites were removed from the analysis.

Figure 4 shows the comparison of data collection between the 3 years. Note that in this figure, 2003 represents the PDA data collection year. As can be seen in this figure, the amount of data collected was very similar for 
Table 1

Comparison of PDA and Paper Data Collection Methods

\begin{tabular}{|c|c|c|c|c|c|c|c|}
\hline \multicolumn{8}{|c|}{ With $\chi^{2}$ Results } \\
\hline & \multicolumn{2}{|c|}{ PDA } & \multicolumn{2}{|c|}{ Paper } & \multirow[b]{2}{*}{$\chi^{2}$} & \multirow[b]{2}{*}{$d f$} & \multirow[b]{2}{*}{ Probability } \\
\hline & $\%$ & $\bar{N}$ & $\%$ & $N$ & & & \\
\hline \multicolumn{8}{|l|}{ Safety belt use } \\
\hline Belted & 79.3 & 973 & 82.1 & 895 & 2.919 & 1 & .088 \\
\hline Not belted & 20.7 & 254 & 17.9 & 195 & & & \\
\hline \multicolumn{8}{|l|}{ Sex } \\
\hline Male & 52.9 & 649 & 55.6 & 606 & 1.754 & 1 & .185 \\
\hline Female & 47.2 & 579 & 44.4 & 484 & & & \\
\hline \multicolumn{8}{|l|}{ Age group } \\
\hline $16-29$ & 36.4 & 428 & 36.0 & 385 & 5.717 & 2 & .057 \\
\hline $30-59$ & 53.3 & 627 & 50.4 & 539 & & & \\
\hline $60+$ & 10.4 & 122 & 13.6 & 145 & & & \\
\hline \multicolumn{8}{|l|}{ Vehicle type } \\
\hline Passenger car & 58.2 & 542 & 60.1 & 511 & 2.362 & 3 & .501 \\
\hline Van/minivan & 14.8 & 138 & 12.5 & 106 & & & \\
\hline SUV & 16.4 & 153 & 16.0 & 136 & & & \\
\hline Pickup truck & 10.6 & 99 & 11.4 & 97 & & & \\
\hline
\end{tabular}

all 3 years. It should also be noted that this particular researcher had had about 5 years of experience collecting data with paper and pencil and that this was his first experience with a PDA. We conclude from these data that data collection with the PDA method is at least as fast as that with the paper-and-pencil method.

\section{DISCUSSION}

Before changing from the traditional paper-and-pencil method of data collection to PDA use, it was important to test this new method to ensure comparability. If the PDA method is not tested and a change in safety belt use or volume of data is noted using the new method, it would not be possible to determine whether the observed change is real or due to a confounding effect or bias related to the use of PDAs. To address these concerns, three areas were assessed during the study: accuracy, speed (volume), and mechanical/environmental issues.
To assess accuracy, the relative contribution of each variable to its category was analyzed. By using this method, it was possible to compare the two data collection methods while eliminating any confounding effects related to differences in the natural speed at which the two comparison researchers collected data. Within each variable analyzed in the study, the relative contributions were not significantly different from one another when the two data collection methods were compared. This result shows that the accuracy of data collection is not adversely affected when the PDA method is used. In other words, using a PDA for safety belt data collection does not in itself change the data collection results. As was mentioned earlier, this is the most important aspect to assess in a study of this type.

Another important aspect to test was the speed of data entry with the new methodology. To assess this issue, a proxy for speed (volume of data compared across survey years) was used. Since data were collected by the same

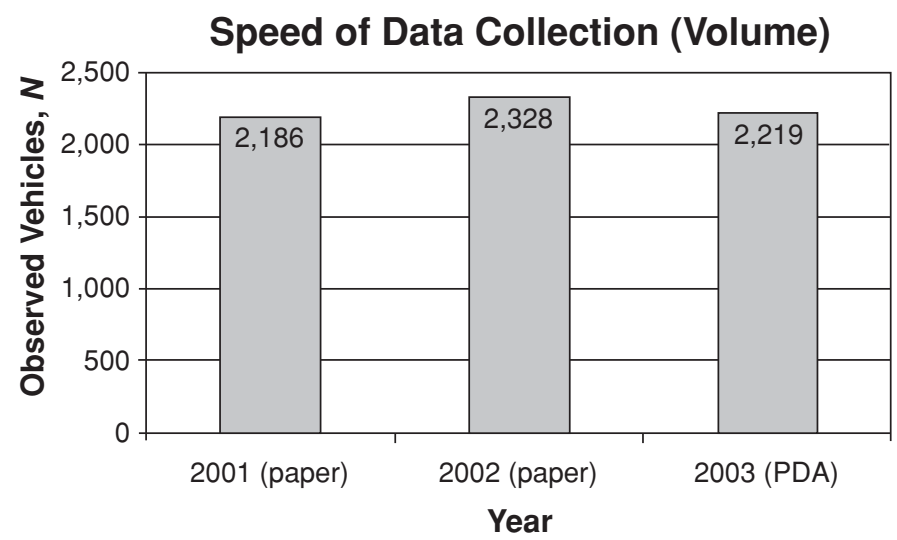

Figure 4. Comparison of the speed (volume) of data collection between the personal digital assistant (PDA) and the paper-and-pencil methods. 
researcher at the same time of day, day of week, and time of year in all three surveys, a direct comparison of the two methodologies was possible. The volume of data collected by the PDA researcher was found to be nearly identical across the surveys. In fact, the amount of data collected by the PDA researcher was about the average of the amounts for the two preceding years. Speed of data collection by the PDA researcher was directly related to the data entry form design. Before the study began, several different designs including different types of input (drop-down lists, text/typing input) were found to be too slow. After many preliminary tests and redesigns, the list and tab, stylus input was chosen. Although speed of data entry is less important than accuracy, significantly fewer data would have resulted in higher variances in the data. The results of this test show that the PDA data collection method will likely be equal to or faster than the traditional method. The anecdotal results concerning environmental issues (which will be discussed in detail later) also suggest that the new method will increase speed during inclement weather.

Mechanical and environmental issues related to PDA use were also tested during the study. Although accuracy and speed were more important to the scientific integrity of the study, the mechanical and environmental issues were equally important to the practical aspects of the PDA methodology. For example, if the PDA battery life lasted only a couple of hours or if the PDA could not be used during rainy weather, this would not be a viable method of field data collection. These aspects of the study were assessed by the PDA researcher and the field supervisor throughout everyday use. Contingencies to address any problems with battery life, data storage capacity, wet weather, and PDA failure/breakage were developed and proved to be effective.

We found that battery life throughout each day was not a problem, since the researcher charged the PDA every evening before data collection. The PDA researcher collected information from 3,294 vehicles during the study. All of this information required only $2.53 \mathrm{MB}$ of storage space. Since this was much less than the $64 \mathrm{MB}$ available for data storage, we experienced no problems with storage capacity or lost data.

During rainy conditions, data could still be easily entered by tapping on the PDA's screen in the appropriate area through the plastic bag. Conversely, with the paperand-pencil data collection method, it was necessary for researchers to carry a large golf umbrella to cover the paper and clipboard or to cover the paper with a large plastic bag during rain or snow. These weather-related advantages were cited by the PDA researcher as perhaps the most positive improvement related to PDA data collection.

Although we purchased an additional, backup PDA and provided the PDA researcher with paper forms as contingencies for PDA failure, it was not necessary to implement either of these contingency plans during the study. The PDA researcher dropped the PDA several times, but it still remained usable. During a debriefing meeting regarding the study, it was determined that the cause of dropping the PDA was related to the use of a hands-free phone sys- tem with the PDA. The researcher liked to leave the handsfree system plugged into the PDA and clipped to his safety vest, in case of a call from the project supervisor. After driving to another location (with the hands-free system still attached in this way), the researcher exited the car and forgot that the system was still attached. This pulled the PDA from the car and caused it to fall to the ground. To eliminate this problem for future studies, plug-in flip covers were purchased for the PDAs, and researchers were told not to leave the hands-free phone systems attached in this way.

Concerns at the study outset regarding screen visibility during different lighting conditions were unfounded. General ease of use was rated by the researcher as much better than that for the paper-and-pencil method in every regard. On the basis of suggestions from the PDA researcher as the study progressed, the electronic forms were altered slightly (i.e., bigger font). The researcher preferred the PDA to paper in every regard; it was smaller and lighter, and the researcher could use it more easily in inclement weather.

Although the e-mail data transfer used with the PDA was far superior to the method used for paper-and-pencil data transfer, a few minor problems were noted. When the researcher traveled outside of the wireless service provider's home network (a very small number of sites), it was not possible to send e-mail. The researcher also noted that sometimes, while e-mailing data, the connection would be lost, and it would become necessary to reset the PDA and try sending the data again. These problems are directly related to shortcomings with the wireless service provider and are, therefore, not possible to overcome. However, several other advantages were also noted. For example, some of our sites were located several hours from the university. However, with the e-mail capability, the researcher could send data to the project supervisor directly from the field, without the need to physically return to the university or even wait until the end of the day. This quick turnaround allowed the supervisor to check data for any errors or problems and to contact the researcher while still in the area to rectify problems. This capability also allowed project staff to access safety belt use rates at any point during the study, making it possible to have a cumulative belt use rate at any given time during the study, instead of waiting until all data collection had been completed and electronically entered.

As the study progressed, and in a subsequent safety belt study, several additional advantages of PDA data collection that were not identified as assessment areas were noted. For example, in a cold weather state such as Michigan, it is difficult to collect data in the winter with the paperand-pencil method. In order to mark the data forms legibly and quickly, researchers could only wear thin gloves on their writing hand. Conversely, with the PDA, researchers could input data accurately and quickly while wearing a ski glove or even a mitten. It was also noted that all the data collected using the PDA had a time/date stamp on it. This provided the field supervisor with additional information by which to ensure quality data collection. Finally, there was a reduction in missing data when the PDA was 
used, in comparison with the paper-and-pencil method. Anecdotally, the researcher noted that moving through the screens from driver to passenger to vehicle and becoming accustomed to how a choice appears as a highlighted bar on the screen made it difficult to forget to mark data.

Before the study began, estimates were also made to determine the cost effectiveness of the PDA data collection method, in comparison with the paper-and-pencil method. We found that researcher training for the new method increased the training period only by $1-2 \mathrm{~h}$. The other costs of implementing the PDA method consist of actual equipment and software fees. These expenses must be compared with costs that are mitigated by the new method - essentially, costs for copying paper forms, along with data entry fees. The analysis of these costs in Michigan revealed that the two methods were nearly equal. Although we recognize that these expenses may vary from state to state, depending on various issues (i.e., method of existing data entry, number of vehicles included per sheet of paper, number of keystrokes per vehicle, etc.), it should also be noted that many of the costs associated with the PDA method are one-time fees. Over time, PDAs may need to be replaced, but this certainly would not be the case for every wave of data collection. Conversely, human data entry costs for paper data would be encumbered with every survey wave, and these fees would likely increase over time. SD cards and software can also be transferred from one PDA to the next, with no added cost. In addition, although we developed and utilized the data entry forms on state-of-the-art PDAs, running the latest version of Palm software, we also tested the programs on much older PDAs, using a much older version of Palm software. We experienced no problems with this older combination and, thus, conclude that it is possible to keep the same equipment for quite some time.

One additional cost that must also be considered is the fee for cellular and wireless Internet service. Although the cost and service contract requirements vary widely by provider and receiving organization, in our study we still found the PDA method (including cellular and wireless Internet service) to be cost effective, since the equipment costs were one-time fees, as was mentioned earlier. However, it is possible to use the PDAs for data collection without the cellular and wireless Internet service. In this case, data would be transferred using SD cards or other inperson methods, rather than e-mailing data. Although this method would save money and still eliminate the cost and time required for electronic data entry, it obviously would lose some of the advantages described earlier.

On the basis of these results, we find that the PDA method of data collection is a cost-effective alternative method of field data collection that is either equal to or better than the paper-and-pencil method in every category tested. In some cases, PDA use was the far superior method. This method provides accurate, fast, and easy data entry for field researchers in all weather conditions.
PDA data collection also eliminates the time-consuming and possibly error-biased process of human data entry of paper forms. No adverse effects on accuracy or speed were observed when the two methods were compared. We conclude that this method of safety belt data collection should be employed as a replacement for the traditional paperand-pencil method. The positive results of this study also suggest that PDAs could be very valuable tools for many other types of field data collection.

\section{REFERENCES}

Bondy, N., \& Glassbrenner, D. (2001). Research note: National Occupant Protection Use Survey-2000 controlled intersection study (Rep. DOT HS 809 318). Washington, DC: U.S. Department of Transportation.

Eby, D. W., Molnar, L. J., \& OlK, M. L. (2000). Trends in driver and front-right passenger safety belt use in Michigan: 1984-1998. Accident Analysis \& Prevention, 32, 837-843.

Eby, D. W., Vivoda, J. M., \& Fordyce, T. A. (2002). The effects of standard enforcement on Michigan safety belt use. Accident Analysis \& Prevention, 34, 101-109.

Glassbrenner, D. (2003). Safety belt use in 2003 (Rep. DOT HS 809 646). Washington, DC: U.S. Department of Transportation.

KIM, K. (1999). The lie factor in traffic safety: Comparison of police and hospital reporting of seat belt and alcohol use in Hawaii. Transportation Research Record, 1665, 141-146.

KIm, S., \& Kıм, K. (2003). Personal, temporal and spatial characteristics of seriously injured crash-involved seat belt non-users in Hawaii. Accident Analysis \& Prevention, 35, 121-130.

National Highway Traffic Safety Administration (1998). Uniform criteria for state observational surveys of seat belt use (Rep. NHTSA98-4280). Washington, DC: U.S. Department of Transportation.

National Highway Traffic SAFEty Administration (2001). Research note: Observed Safety Belt Use Fall 2000 National Occupant Protection Use Survey. Washington, DC: U.S. Department of Transportation.

National Highway Traffic Safety Administration (2002). Traffic safety facts 2002: Overview (Rep. DOT HS 809 612). Washington, DC: U.S. Department of Transportation.

Streff, F. M., \& WagenaAR, A. C. (1989). Are there really shortcuts? Estimating seat belt use with self-report measures. Accident Analysis \& Prevention, 21, 509-516.

YAU, K. K. W. (2004). Risk factors affecting the severity of single vehicle traffic accidents in Hong Kong. Accident Analysis \& Prevention, 36, 333-340.

\section{NOTES}

1. To download the forms created for our project, please visit www .umich.edu/ jviv/safetybelt/PDASafetyBelt.html. These applets (data entry forms) are provided at no cost, and you may change them to meet your needs. However, you must purchase a license for HanDBase (www .handbase.com) to use the program.

2. The PDA traffic-counting method was compared with a mechanical counter before the study began, and no difference was found between the two methods.

3. During the training process, researchers were paired together and collected data independently on the same 25 vehicles. Data from each pair of researchers were then compared for consistency. This process was repeated until the researchers achieved an agreement rate of at least 85\% on each category of data collected.

4. There are four researcher schedules during the annual survey; each collects about one fourth of the data.

(Manuscript received August 3, 2004; revision accepted for publication February 7, 2005.) 